\title{
SOTALOL CHIRAL SEPARATION BY CAPILLARY ELECTROPHORESIS
}

\author{
GABRIEL HANCU ${ }^{*}$, CLAUDIA SĂMĂRGIŢAN ${ }^{1}$, AURA RUSU ${ }^{1}$, ELEONORA MIRCIA ${ }^{2}$ \\ ${ }^{1}$ Department of Pharmaceutical Chemistry, Faculty of Pharmacy, University of Medicine and Pharmacy, Târgu Mureş, Romania; \\ ${ }^{2}$ Department of Organic Chemistry, Faculty of Pharmacy, University of Medicine and Pharmacy, Târgu Mureş, Romania;
}

\begin{abstract}
Differences between the pharmaceutical activity among the enantiomers of sotalol are well known, as R-sotalol and S-sotalol have similar antiarrythmic activities but only the R-enantiomer exhibits $\beta$-blocking activity. In this study capillary zone electrophoresis was used for the enantiomeric separation of sotalol using different native and derivatized; neutral and charged; cyclodextrines as chiral selectors. The effects of $\mathrm{pH}$ value and composition of the background electrolyte, capillary temperature, running voltage and injection parameters have been investigated. The results showed that only the randomly methylated $\beta$-cyclodextrine gave a baseline enantiomeric separation under the optimized conditions; chiral interactions being observed also for hydroxypropyl- $\beta$-CD and sulfobutyl ether- $\beta$ CD. The analytical parameters of the optimized method were verified and the migration order of the two enantiomers was established.
\end{abstract}

\section{INTRODUCTION}

Sotalol (N-4-[1-hydroxi-2-[(1-methyl-ethyl)-amino]-ethyl]-metansulfonamide) is a hydrophilic non-selective $\beta$-blocker officinal in the European Pharmacopoeia $7^{\text {th }}$ edition $(\mathrm{Ph} . E u r .7)^{1}$, characterized more often as a class III antiarrythmic agent (although it has also effects which can be related to class II antiarrythmics as $\beta$-blockers usually do). ${ }^{2}$

It is officinal in the Ph.Eur.7 and used in therapy as sotalol hydrochloride. ${ }^{1,3}$

It contains a chiral center but is marketed as a racemic mixture. The chemical structure of sotalol is presented in figure $\mathbf{1}$.

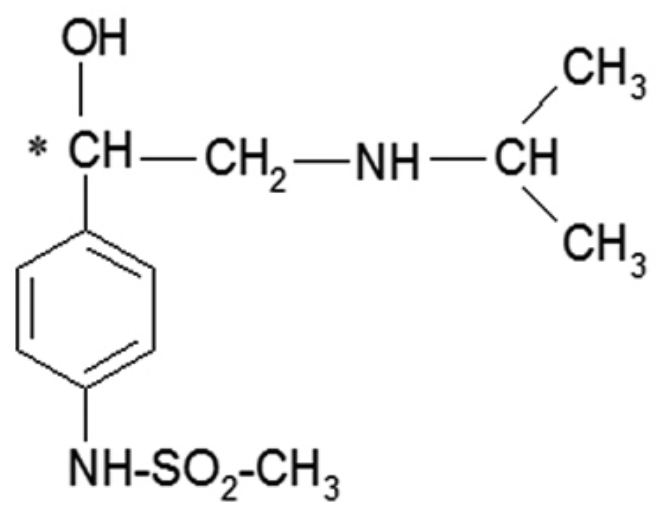

Figure 1 The chemical structure of sotalol. The asterix denote the chiral center.

All $\beta$-blockers used in clinical practice contain an asymmetric carbon atom, and their majority have an aryloxypropanolamine structure. For this type of compounds the levorotatory 1(-)-enantiomer show an S configuration while the dextrarotatory $\mathrm{d}(+)$-enantiomer show an $\mathrm{R}$ configuration. $\mathrm{S}$ enantiomers usually are orders of magnitude more potent in blocking $\beta$-adrenergic receptors in comparison with the respective $\mathrm{R}$ enantiomers. ${ }^{4}$

An exception to this rule is sotalol, which has a phenyl-ethanol-amine structure and its asymmetric carbon atom is located in an ethanolamine type side chain. In this case the priority rules of the four substituents and consequently the absolute configuration of the stereogenic center changes so that R-sotalol (equivalent to l-sotalol) is a much more potent $\beta$-blocker than S-sotalol (equivalent to d-sotalol). ${ }^{4,5}$

Precisely because of its enantioselectivity sotalol mechanism of action is divided between two antiarrythmic drug classes. ${ }^{2}$

The $\mathrm{R}(-)$ enantiomer of sotalol has both $\beta$-blocking (class II antiarrythmic agent) and potassium channel blocking activity (class III antiarrythmic agent), while the $\mathrm{S}(+)$ enantiomer has potassium channel blocking activity and its affinity towards b-adrenergic receptor is 30-60 times lower. Consequently sotalol enantiomers produce different effects on the heart. The action of $\mathrm{S}(+)$ sotalol is associated with the slowing of the sinus heart rate, whereas the action of $\mathrm{R}(-)$ sotalol through its $\beta$-blocking effect contributes to the decrease in heart rate. Overall sotalol prolongs the duration of the action potential, thus increases the effective refractory period of myocardial tissue. ${ }^{2,3,5}$

Sotalol is used in the management of ventricular and supraventricular arrythmias; but because of its proarrhythmic effects, it is usually administered only in severe life-threatening arrhythmias. It was formerly used for its $\beta$-blocking effects in the treatment of hypertension or angina pectoris, but it is not longer recommended for these indications because of the risk of precipitating arrhythmias. ${ }^{3}$

A SWORD (Survival with oral d-sotalol) study investigating the influence of $\mathrm{S}(+)$ sotalol was published with surprising results, as the optically active $\mathrm{S}(+)$ sotalol bare of its $\beta$-blocking effects, increased mortality in patients with myocardial infarction by $65 \%$ compared with placebo; emphasizing even more the potential hazard that might be generated by the administration of a $\beta$-blocking agent distomer. Taking in consideration these aspects one may assume that optically active R(-) sotalol might be possibly more effective in preventing death after myocardial infarction than the racemic mixture which is currently in use, although it consists of $50 \%$ of a drug which is known to be potentially harmful, namely $\mathrm{S}(+)$ sotalol. In the case of sotalol it might appear more reasonable to investigate the enantiomer with $\beta$-blocking effects $\mathrm{R}(-)$ sotalol rather than $\mathrm{S}(+)$ sotalol which lacks the b-blocking effect. ${ }^{6}$

Capillary electrophoresis (CE) is an officinal method of separation in the Ph.Eur. $7^{1}$, comprising a family of related techniques that is based on migration of charged particles dissolved or spread in an electrolyte solution and subdued to the action of an electrical field, which generates electroosmotic and electrophoretic flow of the buffer solution and ionic analytes, within the capillary.

The advantages of using $\mathrm{CE}$ for the enantioseparation of chiral substances are being related with its: high resolution power, simple and rapid method development, low consumption of solvent, sample and chiral selector and especially with the high selectivity in choosing and changing the chiral selector. In CE the chiral selector can be added directly to the buffer solution, selectivity being based on the differential interaction of the enantiomers with the chiral selectors. ${ }^{7,8}$ There are a large variety of chiral selectors which can be used in CE including: cyclodextrines (CDs), macrocyclic antibiotics (macrolides, glycopeptides), proteins, crown ethers, polysaccharides, chiral surfactants, metal-amino acid complexes. ${ }^{7,8}$

However, CDs and their derivatives are the most common chiral selectors in $\mathrm{CE}$ as they are relatively inexpensive, provide a fast equilibration of the $\mathrm{CD}$ analyte complex and offer high separation efficiency and good peak symmetry. ${ }^{8}$

Although through different mechanisms dependent on the individual chiral selectors, chiral resolution results from stereospecific interactions of the selector molecules displaying different affinities towards the two enantiomers of the analytes; giving rise to differences in the respective migration velocities under the applied electric field. ${ }^{8}$

$\mathrm{CE}$ has gained momentum in the achiral and chiral analysis of $\beta$-blockers being regarded today as an alternative and also a complementary method 
to high performance liquid chromatography (HPLC), the most universally accepted method in pharmaceutical analysis.

Several $\beta$-blocker derivatives including sotalol have been determined using capillary zone electrophoresis (CZE) and its associated technique micellar electrokinetic chromatography (MEKC).,10,11

Also chiral separation of sotalol has been studied previously using CZE and CDs derivatives as chiral selectors, the optimal separation conditions significantly differ in several cases, and the basis of the differences has not been clearly identified. ${ }^{12,13}$

Taking in consideration all these aspects regarding the strong connections between chirality and therapeutic use of sotalol, elaboration of new methods for its enantiomers separation become a necessity but also a challenge.

This study describes a screening of various native and derivatized cyclodextrines in order to elaborate a simple, rapid and efficient method for the chiral separation of sotalol enantiomers and the optimization of electrophoretic conditions in order to obtain an enhanced chiral resolution and short analysis time.

\section{MATERIALS AND METHODS}

\section{Chemicals and reagents}

R,S-sotalol hydrochloride, R-sotalol hydrochloride and S-sotalol hydrochloride of pharmaceutical grade were purchased from Moehs Productos Quimicos (Barcelona, Spain). S-propranolol hydrochloride was used as internal standard and was purchased also from Moehs Productos Quimicos (Barcelona, Spain). The commercial preparation of sotalol was Darob (Knoll, Germany) containing $80 \mathrm{mg}$ sotalol hydrochloride. The following reagents of analytical grade were used: phosphoric acid (Pernix Pharma, Hungary), methanol, sodium hydroxide (Lach Ner, Czech Republic), sodium tetraborate, disodium hydrogenphosphate, sodium dihydrogenphosphate (Merck, Germany). Purified water was provided by a Milli-Q Plus water purification system (Millipore, USA).

As chiral selectors we used the following $C D$ derivatives of research grade: native neutral $\mathrm{CDs}(\alpha-\mathrm{CD}, \beta-\mathrm{CD}, \gamma-\mathrm{CD})$, derivatized neutral $\mathrm{CDs}$ (hydroxypropyl- $\beta-\mathrm{CD}-\mathrm{HP}-\beta-\mathrm{CD}$, randomly methylated $\beta-\mathrm{CD}-\mathrm{RAMEB}$ ), anionic substituted charged CDs (carboxymethyl- $\beta-C D$ - CM- $\beta-C D$, sulfobutyl ether- $\beta$-CD - SBE- $\beta$-CD). All CDs were obtained from Cyclolab (Budapest, Hungary) with the exception of SBE- $\beta-C D$ - Capsitol (Cydex, USA).

\section{Equipment}

The experiments were made on an Agilent $6100 \mathrm{CE}$ system (Agilent, Germany) equipped with a diode array UV detector. Separations were performed on a $48 \mathrm{~cm}$ length ( $40 \mathrm{~cm}$ effective length) x $50 \mathrm{~mm}$ I.D uncoated fused silica-capillaries (Agilent, Germany). The electropherograms were recorded and processed by Chemstation 7.01 software (Agilent, Germany). The $\mathrm{pH}$ of the buffer solutions was determined with a Terminal $740 \mathrm{pH}$-meter (Inolab, Germany).

\section{Sample preparation}

Sotalol hydrochloride sample stock solutions were prepared by dissolving the substance in pure water in a concentration of $100 \mathrm{mg} / \mathrm{mL}$ and later diluted to the appropriate concentration. The samples were introduced in the system at the anodic end of the capillary by hydrodynamic injection. All samples and buffers were filtered through a $0.45 \mu \mathrm{m}$ syringe filter and degassed by ultrasound for 5 minutes before use. The capillaries were conditioned before use with $0.1 \mathrm{M}$ sodium hydroxide for 30 minutes and with the background electrolyte used in the analysis for 30 minutes. The capillary was rinsed for 1 minute with $0.1 \mathrm{M}$ sodium hydroxide and buffer solutions before each electrophoretic determination.

To determine sotalol from Darob tablets, twenty tablets from the same batch product (each containing $80 \mathrm{mg}$ sotalol hydrochloride) were weight and pulverized in a mortar, and an amount of powder equivalent to the average weight of a tablet was accurately weighed and used. The powder was dissolved in water, and then the solution was diluted to the appropriate concentration, sonicated for 10 minutes and filtered through $0.45 \mu \mathrm{m}$ syringe filter. The samples was centrifuged at $3500 \mathrm{rpm}$ for 10 minutes, the supernatant was diluted; further the same procedures were followed as for the preparation of standard solutions for the CE separation.

\section{Evaluation of separation}

The separation factors (a) were calculated as the ratio of the migration times of the optical isomers, and the resolution $(\mathrm{R})$ was obtained by the $\mathrm{R}=2\left(\mathrm{t}_{2}\right.$ $\left.-t_{1}\right) /\left(w_{1}+w_{2}\right)$ equation, where the migration times $\left(t_{1}\right.$ and $\left.t_{2}\right)$ and the peakwidths at the baseline $\left(\mathrm{w}_{1}\right.$ and $\left.\mathrm{w}_{2}\right)$ were marked for the slow and fast migrating enantiomers, respectively.
We also evaluated the migration retardation factor $\left(\mathrm{R}_{\mathrm{m}}\right)$ defined as the ratio of the migration time of the analyte (if resolved, of the second eluted enantiomer) in the buffer containing the chiral selector and the migration time of the analyte in the plane phosphate buffer; which represents an approximate parameter for measuring the strength of the host-guest interaction.

\section{RESULTS AND DISCUSSION}

\section{Preliminary analysis}

Electrophoretic mobilities and ionization behavior of analytes are the key factors driving separations in CZE. Knowledge of these basic physicochemical properties of analytes gives valuable information about their nature and makes it easier to choose appropriate experimental conditions for their separation.

In order to find the suitable conditions for the chiral separation of sotalol, a series of preliminary experiments were conducted at different $\mathrm{pH}$ values and buffer compositions. In the preliminary analysis we used: $25 \mathrm{mM}$ phosphoric acid $(\mathrm{pH}-2.1), 25 \mathrm{mM}$ disodium hydrogenphosphate $-25 \mathrm{mM}$ sodium dihydrogenphosphate $(\mathrm{pH}-7)$ and $25 \mathrm{mM}$ sodium tetraborate $(\mathrm{pH}-9.3)$ background electrolytes respectively and we modified the $\mathrm{pH}$ of the buffer by adding a $0.1 \mathrm{M}$ sodium hydroxide solution.

We recorded previously the UV spectra of sotalol and found its absorption maximum at $232 \mathrm{~nm}$, which was selected as detection wavelength in the $\mathrm{CE}$ separations. We applied some "standard" electrophoretic conditions for a CZE analysis: temperature $20{ }^{\circ} \mathrm{C}$, applied voltage $+20 \mathrm{kV}$, injection pressure/time $50 \mathrm{mbar} / 3 \mathrm{sec}$, sample concentration $10 \mu \mathrm{g} / \mathrm{mL}$. Sotalol has two $\mathrm{pKa}$ values, one $(\mathrm{pKa}-8.3)$ corresponding to the sulfonamide substituent while the other (pKa - 9.8) corresponds to the amine group; consequently an acidic solution should be a suitable background electrolyte for the separation.

As expected, the most suitable background electrolyte was a $25 \mathrm{mM}$ phosphoric acid solution at a $\mathrm{pH}$ of 2.5. A well-shaped peak appeared for sotalol using this buffer at 8.1 minutes (migration time). At this very low $\mathrm{pH}$ the influence of electroosmotic flow (EOF) is minimal, and the analyte will migrate mostly through its own electrophoretic mobility.

The difficulties in separating sotalol enantiomers in comparison with other $\beta$-blockers reported previously in the literature could be related to the particular structural characteristics of this compound, which is somewhat different from that of other $\beta$-blockers. ${ }^{12}$

\section{Optimization of analytical conditions}

Initial concentration of $10 \mathrm{mM}$ neutral $\mathrm{CDs}$ were added to the buffer solution, while for charged CDs we added a concentration of $5 \mathrm{mM}$ in order to limit the increase of ionic strength which generated high currents.

No chiral interactions were observed when using native neutral CDs $(\alpha-$ $\mathrm{CD}, \beta-\mathrm{CD}, \gamma-\mathrm{CD})$, as we observed only an increase in migration times of the analyte.

When using derivatized neutral CDs (HP- $\beta$-CD, RAMEB) stereoselective interactions were observed even with the initial electrophoretic parameters; a slight peak splitting for HP- $\beta$-CD and a chiral resolution of 0.77 for RAMEB.

It was demonstrated experimentally that charged $\mathrm{CD}$ are more effective chiral selectors than the neutral ones for a large numbers of $\beta$-blockers, because of the presence of additional ionic interactions that contribute to the chiral recognition At this low $\mathrm{pH}$, the anionic charged SBE- $\beta-\mathrm{CD}$ is negatively charged while sotalol is positively charged, so the SBE- $\beta-C D$ will move towards the anode while sotalol enantiomers will move towards the cathode. ${ }^{14,15}$

SBE- $\beta$-CD will display a migration opposite to that of the enantiomers, which resulted in a significant increase of the migration times (around 25 minutes) but to a rather low chiral resolution $(\mathrm{R}-0.7)$. Another anionic charged $\mathrm{CD}, \mathrm{CM}-\beta-\mathrm{CD}$ led also to high migration times (around 20 minutes) but no chiral resolution.

The use of a dual CD system, which include a combination of a neutral and a charged CD (10 mM $\beta-C D+5 \mathrm{mM} \mathrm{SBE}-\beta-\mathrm{CD})$ led to an increase of migration times but only a small peak splitting has been observed.

After this initial CD screening we selected as the optimal chiral selector the randomly methylated $\beta-C D$ (RAMEB). Increasing the RAMEB concentration led to an increase in the chiral resolution and also in the migration times of the enantiomers; a concentration of $30 \mathrm{mM}$ RAMEB giving baseline separation of the two enantiomers. While the RAMEB concentration increased from 10 to $30 \mathrm{mM}$, the chiral separation improved greatly, whereas the concentration was increased in the range $30-50 \mathrm{mM}$, only little influence on the separation could be observed.

The optimum concentration depends on the binding affinity of stereoisomers with the chiral selector. At a low CD concentration, no separation of the enantiomers is possible because there are not enough chiral selectors available 
to form the complexes, while, at a high concentration, the enantiomers may be completely complexed and do not result in separation. Therefore, it is essential to determine the optimum $\mathrm{CD}$ concentration to obtain the best chiral resolution.

Increasing the $\mathrm{pH}$ value increased slightly the chiral resolution, but the addition of a $0.1 \mathrm{M}$ sodium hydroxide solution to the buffer solution increased the generated current and led to instability of the electrophoretic system. Consequently we chose a $\mathrm{pH}$ of 2.5 .

Also at low $\mathrm{pH}$ values there is more time for the analyte to interact with the $\mathrm{CD}$ to result in increased time spent in the capillary, while at higher $\mathrm{pH}$, the analyte eluted faster due to increasing EOF, with EOF becoming more significant at a $\mathrm{pH}$ value around 5 .

An increase of the buffer concentration led to an increase of the migration times, but had no marked effect on the separation resolution. Addition of an organic modifier such as methanol or acetonitrile to the phosphate buffer resulted in longer migration times but there was no significant improvement in the chiral resolution.

While the applied voltage had only a slight influence on the chiral resolution, temperature influenced strongly the separation efficiency and the enantiomeric resolution, as a decrease in temperature led to longer migration times but also to an increased chiral resolution. The effect of capillary temperature on chiral separation is related to the thermodynamic behavior of the analyte-chiral selector complex during the chiral separation. In order to obtain a satisfactory

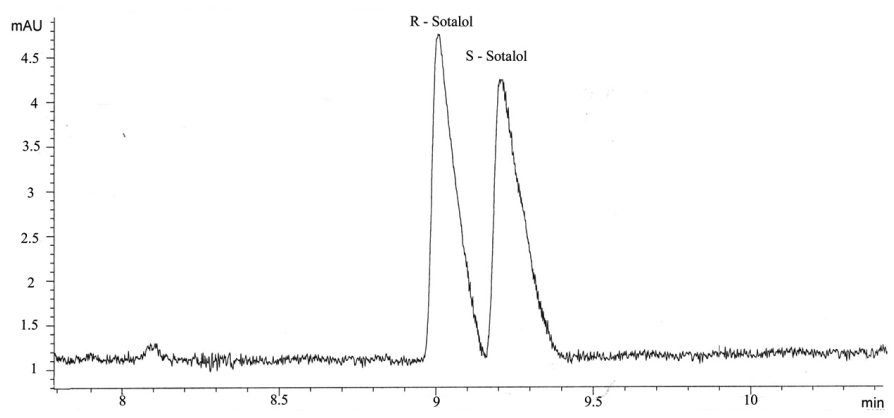

Figure 2 Capillary electrophoretic separation of sotalol enantiomers (experimental conditions: BGE - $25 \mathrm{mM}$ phosphoric acid + $30 \mathrm{mM}$ RAMEB, $\mathrm{pH}-2.5$, voltage $+25 \mathrm{kV}$, temperature $15^{\circ} \mathrm{C}$, hydrodynamic injection 50 mbar x 2 sec., sample concentration $10 \mathrm{mg} / \mathrm{ml}$, UV detection $232 \mathrm{~nm}$ ) migration times and improve chiral resolution we combined the effects of these two secondary electrophoretic parameters, choosing an applied voltage of +25 $\mathrm{kV}$ at a temperature of $15^{\circ} \mathrm{C}$.

A high injection pressure and a short injection time will increase chiral resolution; in order to obtain a quantifiable electrophoretic response and improve enantiomeric resolution we chose an injection pressure of $50 \mathrm{mbar}$ for 2 seconds.

The migration order of the two enantiomers was determined by spiking and also by injecting the pure enantiomers separately using the selected chiral buffer background electrolyte.

Taking in consideration the aspects presented above we can conclude that the optimal electrophoretic conditions for the sotalol enantioseparation are: 25 $\mathrm{mM}$ phosphoric acid background electrolyte, $30 \mathrm{mM}$ RAMEB chiral selector, buffer $\mathrm{pH} 2.5$, applied voltage $+25 \mathrm{kV}$, temperature $15^{\circ} \mathrm{C}$, injection pressure/ time $50 \mathrm{mbar} / 2 \mathrm{sec}$. Applying the optimized electrophoretic conditions we succeeded in the separation of the two enantiomers in less than 10 minutes, with a chiral resolution of 1.39 and a separation factor of 1.04, the order of migration being R-sotalol followed by S-sotalol (figure 2).

By applying the same electrophoretic conditions but using $20 \mathrm{mM} \mathrm{HP}-\beta-$ $\mathrm{CD}$ as chiral selector we obtained a chiral resolution of 0.73 and a separation factor of 1.03 . Increasing the HP- $\beta-C D$ concentration didn't increase chiral resolution (figure 3).

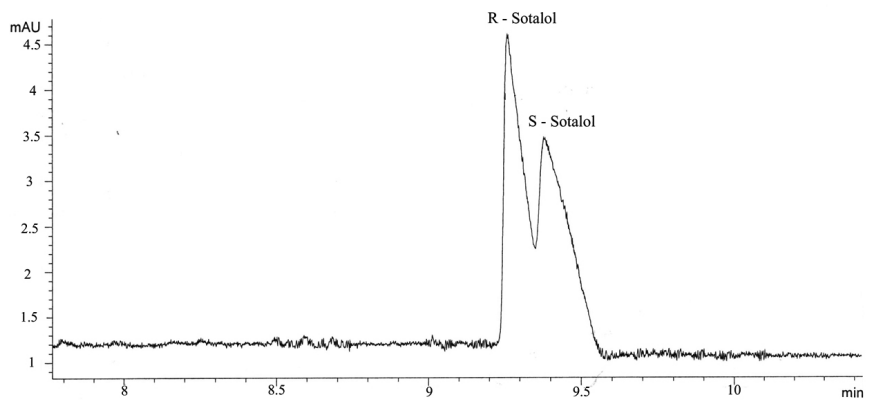

Figure 3 Capillary electrophoretic separation of sotalol enantiomers (experimental conditions: BGE - $25 \mathrm{mM}$ phosphoric acid + $20 \mathrm{mM} \mathrm{HP}-\beta-\mathrm{CD}$ $\mathrm{pH}-2.5$, voltage $+25 \mathrm{kV}$, temperature $15^{\circ} \mathrm{C}$, hydrodynamic injection 50 mbar x 2 sec., sample concentration $10 \mathrm{mg} / \mathrm{ml}$, UV detection $232 \mathrm{~nm}$ )

Table 1 summarizes the optimized separation conditions (concentration and type of selector, pH, voltage, temperature) and results (migration times of enantiomers, migration retardation factor, separation factor, resolution).

Table 1. Capillary electrophoretic separation of optical isomers of sotalol using cyclodextrin derivatives as chiral selectors

\begin{tabular}{|c|c|c|c|c|c|c|c|c|}
\hline CD-derivative & $\mathbf{p H}$ & $\begin{array}{c}\text { Voltage } \\
(\mathbf{k V})\end{array}$ & Temperature $\left({ }^{\circ} \mathbf{C}\right)$ & $\mathbf{t}_{\mathbf{1}}(\mathbf{m i n})$ & $\mathbf{t}_{\mathbf{2}}(\mathbf{m i n})$ & $\mathbf{R}_{\mathbf{m}}$ & $\alpha$ & $\mathbf{R}$ \\
\hline $30 \mathrm{mM}$ RAMEB & 2.5 & 25 & 15 & 9.1 & 9.5 & 1.16 & 1.04 & 1.39 \\
\hline $20 \mathrm{mM}$ HP- $\beta-C D$ & 2.5 & 25 & 15 & 9.3 & 9.6 & 1.17 & 1.03 & 0.73 \\
\hline $5 \mathrm{mM}$ SBE- $\beta-C D$ & 2.5 & 20 & 20 & 24.8 & 25.2 & 3.09 & 1.01 & 0.70 \\
\hline
\end{tabular}

\section{Analytical performance}

The analytical performance of the method was evaluated using the optimized electrophoretic conditions and S-propranolol as internal standard.

The relative standard deviations (RSD) for the migration times and peak areas was calculated by injecting consecutively $(\mathrm{n}=6)$ a sample of $10 \mu \mathrm{g} / \mathrm{ml}(\mathrm{table} 2)$.

Calibration plots were constructed by preparing standard solutions $(n=3)$ at six different concentrations in a specific concentration range (concentration range: $2.5-50 \mu \mathrm{g} / \mathrm{mL}$ ). The regression equation and correlation coefficient are presented in table 3 .

The limits of detection (LOD) and quantification (LOQ) were estimated as: standard deviation of regression equation/slope of the regression equation multiplied by 3.3 and 10 , respectively (table 2 ).

Table 2. Analytical parameters of the sotalol chiral separation

\begin{tabular}{|c|c|c|c|c|c|}
\hline Enantiomer & Migration time (min) & RSD migration time (\%) & RSD peak area (\%) & LOD $(\boldsymbol{\mu g} / \mathbf{m l})$ & $\mathbf{L O Q}(\boldsymbol{\mu g} / \mathbf{m l})$ \\
\hline R-sotalol & 9.03 & 0.15 & 0.83 & 1.13 & 3.45 \\
\hline S-sotalol & 9.53 & 0.23 & 1.17 & 1.25 & 3.75 \\
\hline
\end{tabular}


Table 3. Linearity regression data of the sotalol chiral separation

\begin{tabular}{|c|c|c|}
\hline Enantiomer & Regression equation & $\begin{array}{c}\text { Correlation } \\
\text { coefficient }\end{array}$ \\
\hline R-sotalol & $\mathrm{y}=0.7067 \mathrm{x}+1.5485$ & 0.993 \\
\hline S-sotalol & $\mathrm{y}=0.6284 \mathrm{x}+1.4$ & 0.994 \\
\hline
\end{tabular}

The optimized method was applied to determine sotalol enantiomers from pharmaceutical tablets containing $80 \mathrm{mg}$ sotalol hydrochloride. The peaks obtained from the samples prepared from tablets were similar with those obtained from sotalol standard and there where no noticeable interference from the matrix. Recovery was between 98.5 and $101.5 \%$ for drug substance. The method proved to be suitable for determination of sotalol in tablets, and demonstrate that the developed method can be used in the analysis of true samples.

In order to explain a chiral separation there are three main aspects that should be taken into consideration: the role of the chemical structure of the analyte, the effect of the experimental parameters and the structure of the chiral selector. ${ }^{12,14,15}$

The two CDs (RAMEB and HP- $\beta-C D$ ), which exhibited chiral interactions with sotalol enantiomers are derivatized one; have the advantage of a good solubility in aqueous buffers and also exhibit a better selectivity than the native $\mathrm{CDs}$, but are neutral from electrophoretic point of view. Consequently the migrations of sotalol enantiomers will be towards the cathode while the neutral CDs will almost not move, because the EOF in acidic background electrolytes is close to zero.

The migration velocity of the complexed form will differ from that of the free molecule, because of the bigger size of the complex with the same charge as the free form. ${ }^{8}$

The chiral discrimination depends on the difference of interactions between $C D$ derivative with $\mathrm{R}$ - and $\mathrm{S}$ - enantiomers. The migration time of the two enantiomers indicates the interaction between the selected $\mathrm{CD}$ and the enantiomers of sotalol.

Therefore, the higher the affinity for the $\mathrm{CD}$, the lower the overall electrophoretic mobility of the enantiomer. The faster migration times of the $\mathrm{R}$-enantiomer indicates that this enantiomer has a weaker interaction with the $\mathrm{CDs}$, while the interaction of S-sotalol is stronger. Previous studies involving other $\beta$-blockers reported usually the faster migration of the $S$ enantiomer, but in the case of sotalol the changing in the migration order of the enantiomers only reflects the particularities in its chemical structure. ${ }^{12}$

\section{CONCLUSIONS}

As a consequence of the facts presented above it is clear that the mechanism of action and the therapeutic effects of $\mathrm{R}(-)$ and $\mathrm{S}(+)$ enantiomers of sotalol exhibit a high degree of stereoselectivity. Therefore probably the optically pure enantiomer should be recognized and used in therapy as distinct drugs, as the racemate can no longer be regarded as optimal for patients on sotalol therapy.

$\mathrm{CE}$ offers tremendous flexibility for enantiomeric separations, requiring only the addition of a chiral selector to the buffer solution; CDs and their derivatives remaining the most extensively used chiral additives.

The separation conditions including chiral selector type and concentration, running buffer concentration and $\mathrm{pH}$ and working voltage and temperature were optimized, and the two enantiomers were identified. CD type and concentration, $\mathrm{pH}$ value of the background electrolyte and system temperature had a strong influence on the efficiency of the chiral separation. The changes in the concentration of the cyclodextrins and in the $\mathrm{pH}$ of the background electrolyte showed uneven effect on the resolution of the optical isomers of sotalol Among the analyzed CDs, RAMEB exhibited the best chiral resolution being the only $\mathrm{CD}$ that gave a baseline enantiomeric resolution, chiral interactions being observed also for HP- $\beta-C D$ and SBE- $\beta-C D$.

This CZE method is rapid, specific, reliable and cost effective and can be used by laboratories performing routine chiral analysis of sotalol.

\section{ACKNOWLEDGEMENTS}

Financial support for this study was granted through an Internal Research Grants funded by the University of Medicine and Pharmacy from Tîrgu Mureş, Romania (grant contract for execution of research projects nr.22)

\section{REFERENCES}

1. European Pharmacopoeia, $7^{\text {th }}$ ed. Council of Europe, Strasbourg, 2010.

2. J.H. Block. J.M. Beale (eds.), Wilson and Gisvold's textbook of Organic Medicinal and Pharmaceutical Chemistry. $11^{\text {th }}$ ed. Lippincott Williams\&Wilkins, Philadelphia, 2004, p. 642.

3. Martindale, The Complete Drug Reference, $37^{\text {th }}$ ed. Pharmaceutical Press, London, 2011, p. 1398-1399.

4. K. Stoschitzky, W. Lindner, G. Zernig, J. Clin. Bas. Cardiol. 1(1), 15, (1998).

5. R. Mehvar, D. Brocks, J. Pharm. Pharmaceut. Sci. 4(2), 185, (2001).

6. A.L. Waldo, A.J. Camm, H. deRuyter, P.L. Friedman, D.J. MacNeil, J.F. Pauls, B. Pitt, C.M. Pratt, P.J. Schwartz, E.P. Veltri, Lancet 348, 7, (1996).

7. P. Mikus, Chiral capillary electrophoresis in current pharmaceutical and biomedical analysis, InTech, Rijeka, 2012, p. 5-51.

8. G. Gubitz, M. Schmid (eds.), Chiral separations - Methods and Protocols. Humana Press, Totowa, New Jersey, 2004, p. 255-343.

9. A.E. Bretnall, B.S. Clarke, J. Chromatogr. A 745, 145, (1996).

10. H. Siren, R. Kuldvee, T. Karla, T. Ekstrom, M.L. Riekkola, J. Chromatogr. A 1068, 89, (2005).

11. D. Dogrukol-Ak, A.G. Dal, M. Tuncel, Cromatographia 66, 159, (2007).

12. L. Gagyi, Á. Gyéresi, F. Kilár, J. Biochem. Biophys. Meth. 70, 1268, (2008).

13. L. Suntonsurk, Anal. Bioanal. Chem. 52, 29, (2010).

14. G.S. Yang, D.M. Chen, Y. Yang, B. Tang, J.J. Gao, H.Y. Aboul-Enein, B. Koppenhoefer, Chromatographia 62, 441, (2005).

15. H. Zhang, H. Shao, A. Youmei, Z. Zhang, Chromatographia 68, 653, (2008). 
J. Chil. Chem. Soc., 59, No 3 (2014) 